\title{
AN AUTOMATIC SYSTEM FOR DIRT IN PULP INSPECTION USING HIERARCHICAL IMAGE SEGMENTATION
}

\author{
F. Duarte†t, H. Araújoß, A. Douradoł \\ †CISUC, Dep. de Eng. Informática da Universidade de Coimbra \\ $\S$ Dep. de Eng. Electrotécnica da Universidade de Coimbra \\ $\ddagger$ Instituto Superior de Engenharia de Coimbra, P-3030 Coimbra, PORTUGAL \\ FAX: (+351) 39701266 Email:\{fduarte,dourado\}@dei.uc.pt
}

\begin{abstract}
An automatic visual inspection system designed for dirt inspection in the pulp and paper industry is presented. A new hierarchical region oriented segmentation algorithm is introduced. The algorithm is tuned according to the singular characteristics of the pulp samples. A criterion based on the maximisation of the local contrast is defined in order to perform a defect region segmentation of the pulp and paper images. Some optimisations are introduced to avoid an excessive computational load. (C) 1999 Elsevier Science Ltd. All rights reserved.
\end{abstract}

Keywords: Quality Control; Automatic Visual Inspection; Production; Pulp and Paper; Computer Vision; Pattern Recognition.

\section{INTRODUCTION}

The pulp and paper industry has a rather important role in the Portuguese economy, since it is one of the leading basic sectors. In this industry, most of the quality control is still performed by human visual inspection. The aim of the present work is to replace the human visual inspection by a full automatic dirt counting system, which is able to perform the same quality control and to obtain faster, more objective and more reliable results. The system in intended to on-line inspection, enabling therefore the output information to be used as feedback control to the wood cooking and to the pulp bleaching sections.

Dirt counting is an off line operation carried accordingly to standard procedures, where the human inspector compares each dirt speck with a series of printed dots in a standard chart (a photographic print of a series of black spots of various areas on a white background) and chooses the dot which matches it most closely. This task is made accordingly to the concept of "visual equivalent black area", that relies upon the principle that a large gray speck has the same visual impact as a smaller and a darker speck viewed under the same conditions (Jordan, D. B. and Nguyen., 1985; F. Duarte, 1995). This procedure is very time-consuming, and highly dependant on subjective human judgement, which varies overtime due to fatigue.

The dirt comes from soot, bark, shives, inadequately de-inked stock and various other sources. For quality assessment, dirt is considered any foreign matter embedded in a sheet which exhibits a marked contrast with respect to the rest of the sheet and an equivalent black area equal or greater than of $0.04 \mathrm{~mm}^{2}$.

Localisation of these defects in the image is purely random due to the nature of the pulp and paper formation process. The pulp structure is mainly composed by cellulose fibbers in a random distribution, but contains also several different kinds of particles like water, silica, starch, calcium, rosin, etc., with air separating them. This structure gives a typical rough texture to the pulp, which induces a considerable background noise. Therefore, usually it is observed that the noise intensity is able to disturb the visual perception of some dirt specks, increasing the difficulties for computer vision based detection, namely at the image segmentation level.

0360-8352/99 - see front matter (C) 1999 Elsevier Science Ltd. All rights reserved.

PII: S0360-8352(99)00089-3 
The image acquisition environment is identical to the one used in the traditional inspection. The pulp is placed over a transparent table with back-illumination that gives about $540 \mathrm{~lm} / \mathrm{m}^{2}$ of white light. The pulp sample images are captured from above downward by the CCD video camera, interfaced with a video board.

To solve the described segmentation difficulties a new low-level segmentation algorithm is introduced, based in a hierarchical approach. Other hierarchical segmentation approaches are described in (Beaulieu, J. M. and Goldberg, 1989; Peter F. M. N., 1995), However, those methods are not able to detect properly, very small regions.

This method which is presented in section 2, is tuned for these singular aspects (i.e. small size regions which are statistically negligible with a considerable background noise) in the defect region extraction and classification. First, a hierarchical decomposition of the image will produce a huge region set obtained with a local threshold. These regions are strategically organised in a tree structure, where each node corresponds to one region, as described in section 2.1. The segment classification, in terms of visual impact feature is described in section 2.2. A global criterion is defined in order to perform a region selection based on several region characteristics, such as, the local contrast, the regions size and its localisation in the tree structure as described in section 2.3.

\section{THE HIERARCHICAL IMAGE SEGMENTATION}

This section presents the new hierarchical segmentation algorithm based on the maximisation of the local contrast. The aim of the segmentation process is the detection and classification of the dirt specks presented in the pulp sample. Let $G=\{0,1, \ldots, l-1\}$ be the set of gray levels, where $l$ is the total number of quantification levels. The image function $f$ with gray value at pixel $(x, y)$ is denoted by $f(x, y)$. Let $B=\{b, w\}$ be a pair of gray levels with $b, w \in G$. The result of thresholding the image function $f(x, y)$ at gray level $t$, is a binary image function $f^{t}(x, y)$. The explicit representation of $f^{t}(x, y)$ is expressed as (1)

$$
f^{t}(x, y)= \begin{cases}b, & \text { if } f(x, y) \leq t \\ w, & \text { if } f(x, y)>t\end{cases}
$$

This binary image $f^{t}(x, y)$ presents a white background and $n_{t}$ black regions (set of connected pixels with $b$ value) that we call segments. Each of these regions is a candidate for a real defect in the original image. The segments in the binary image $f^{t}(x, y)$ are represented in the partition set (2)

$$
P^{t}=\left\{S_{1}^{t}, S_{2}^{t}, \cdots, S_{n_{t}}^{t}\right\}
$$

Considering the $l$ binary images produced with all the threshold levels $T=\{0,1, \cdots, l-1\}$, we obtain a generalised segmentation partition set with all the segments, which are present in those binary images:

$$
\Theta=\left\{P^{0}, P^{1}, \cdots, P^{l-1}\right\}=\left\{S_{1}^{0}, S_{2}^{0}, \cdots, S_{n_{0}}^{0}, S_{1}^{1}, S_{2}^{1}, S_{n_{1}}^{1}, \cdots, S_{1}^{l-1}\right\}
$$

This partition set will be the basis of the tree of segments defined in the next section.

\subsection{The Hierarchical Tree of Segment}

The large amount of segments in the partition set $\Theta$ defined in (3) is now strategically organised in a tree structure, where each node corresponds to a segment $S_{i}^{t}$. The tree is obtained by linking segments in adjacent hierarchical levels (parent $\rightarrow$ son), as represented in Fig. 1 .

The tree's root is formed by segment $S_{1}^{l-1}$, which is unique, since it is obtained from the $1-1$ threshold. This segment corresponds to the entire image, since $f(x, y) \leq l-1, \forall(x, y) \in f \Rightarrow P^{l-1}=\left\{S_{1}^{l-1}\right\}$. The tree is further constructed by linking segments at higher hierarchy levels to segments at lower levels, according to fowling constraint:

- A link between a segment $S_{i}^{\tau}$ (parent) and a segment $S_{j}^{\sigma}$ (son) exists, if $\tau=\sigma+1$ and $S_{j}^{\sigma}$ is a disjoint subpart of $S_{i}^{\tau},\left(S_{j}^{\sigma} \subset S_{i}^{\tau}\right)$.

Segment $S_{j}^{\sigma}$ is descendant of the segment $S_{i}^{\tau}$, if there exists a monotonic descendant path, starting at segment $S_{i}^{\tau}$ and ending at segment $S_{j}^{\sigma}\left(S_{j}^{\sigma} \subset S_{i}^{\tau}\right.$ and $\left.\sigma<\tau\right)$. Segment $S_{j}^{\sigma}$ is an ascendant of segment $S_{i}^{\tau}$, if $S_{i}^{\tau}$ is descendent of $S_{j}^{\sigma}$. A segment $S_{j}^{\sigma}$ is a leave if they have not descendants. 
Those trees exhibit some useful properties:

(i) All segments in set $P^{i}$ will be stored in the hierarchical level $i$ of the tree.

(ii) A unique path is defined as starting at one of the leaves and climbing up the tree until the root is reached.

(iii) Except for the root segment $S_{1}^{l-1}$, all the tree segments have exactly one parent.

(iv) The tree structure defined in this section contains all the information of the original image. It is possible to reconstruct the original image without losing information.

After computing the tree, it is necessary to select all those tree segments, corresponding to real defects. This will be described in the next section.

\subsection{The true segment detection}

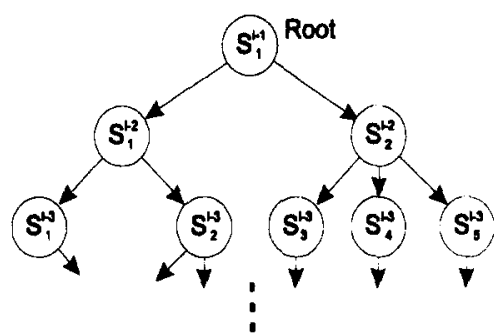

Fig. 1 - The tree of segments

The tree structure defined in the previous section contains a considerable amount of segments (all the segments of the set $\Theta$ ), however only a subset of $\Theta$ has a real significance. We will now present a selection method to pick up only the segments that correspond to real defects in the original image.

Neighbourhood $V_{S}$ of segment $S$ is defined as the region involving the segment, whose area $A_{V}$ is identical to the segment area $A_{S}$ (Fig. 2). The segment contrast $C_{S}$ is defined as

$$
C_{S}=\left|B_{S}-B_{V}\right|
$$

where $B_{S}$ is the median gray level of the tree segment region and $B_{V}$ is the median gray level of the neighbourhood. The median grays levels $B_{S}$ and $B_{V}$ are computed considering the gray level values of the original image $f(x, y)$.

The goal is to obtain prominent segment subset $P^{*}$ that contains only those segments representing the set of real objects in the original image. A segment $S \in P^{f}$ is a true segment if it satisfies the two following constrains:

1. The contrast $C_{S}$ of the segment $S$ is higher or equal than the contrast of any of its descendants.

2. The contrast $C_{S}$ of the segment $S$ is higher than the contrast of any of its ascendants.

Fig 3.b) represents the true segment set $P^{*}$, obtained by the outlined method. It should be noted that the gray level represents the respective segment contrast.

\subsection{The Segment Classification}

In the last step of the segmentation process all the segments belonging to the subset $P^{*}$ will be classified in terms of their "equivalent black area" $A_{S}^{e q}$ which is defined as the area of a very black segment in a white background that exhibits the same visibility as segment $S$. The segment visibility (visual impact) depends on its size and contrast. A tiny dirt speck is more visible if it is very black. The combined effect of contrast and size on visual impact can be approximate by the expression (4)

$$
A_{S}^{e q}=A_{s} \frac{C_{S}}{C_{\max }}
$$

where $C_{\max }$ is the maximum contrast that we can find in a very black dirt speck. This parameter depends on the characteristics of the image acquisition equipment and the illumination environment and its value is fixed in the calibration procedure. A spatial and contrast calibration is performed using a photographic calibration chart with a set of black spots with a maximum contrast and predefined size.

The black squares in the Fig 3c) represents black equivalent size of the dirt specks of the original image (Fig 3a), computed with equation (4). Some true segments do not exhibit enough contrast to be considered as dirt specks. 
Therefore a minimum contrast threshold is introduced for the segments to be considered as real ones. The system is now able to produce an inspection report as described in the next section.

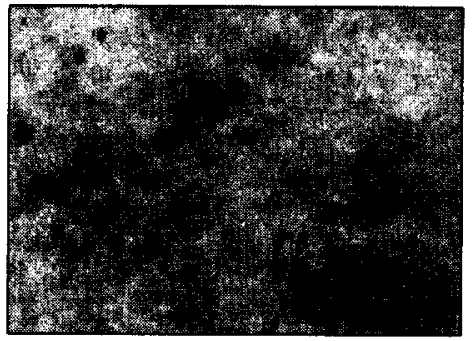

a)

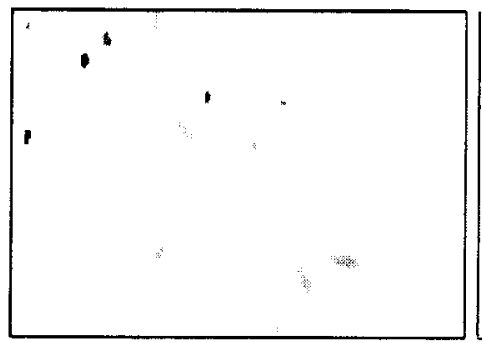

b)

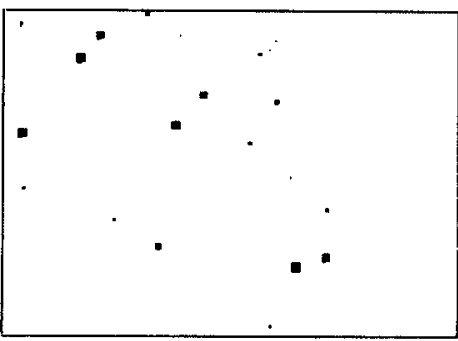

c)

\section{THE INSPECTION REPORT}

In the last stage of the inspection procedure an inspection report will be produced, presenting the following information according to the standard norms (CPPA, 1984):

- The total of pulp area examined.

- The mean dirt speck size detected in terms of square millimiters of equivalent black area of dirt per square meter of surface examined.

- The total of dirt specks detected.

- The total of dirt specks normalizad per one square meter of surface examined.

- A histogram of size distribution.

\section{CONCLUSION}

The major constrain in computer vision based dirt inspection is due to region extraction for measurement. To solve this problem a new hierarchical image segmentation algorithm in low level term was presented. This is the most important aspect of the system design, considering that the quality of the inspection results depends mainly on the correct detection and classification of the dirt specks. This algorithm exhibits a high sensitive response to small particles and simultaneously has a good immunity to the background noise.

A drawback of the direct implementation of this method is its excessive computing time for large region sets in the hierarchical tree structure. Some design techniques are introduced to reduce the computational effort. A method is developed to reduce substantially the tree size before the region classification takes place. For instance, the segments at higher hierarchical levels that correspond to larger regions with a whiten gray tone, do not exhibit a priori conditions to be real segments, so they can be discarded.

It has been shown experimentally that the algorithm can correctly perform the dirt specks detection, by a region search based on local image proprieties, according to a local contrast maximisation criterion. The immunity to the low frequencies noise is due to the local segmentation strategy. As can be observed Fig 3.c), the segmentation algorithm presents a low classification error rate.

Acknowledgements: The authors express their gratitude to Portucel E. P. for the support and for the used pulp samples. This work was partially supported by INIC/FCTUC/PRAXIS XXI.

\section{REFERENCES:}

Beaulieu, J. M. and Goldberg M. (1989), Hierarchy in Picture Segmentation: A Stepwise Optimization Approach, IEEE Transactions on Pattern Analysis and Machine Intelligence, 12, 150-163.

CPPA (1984) Standard Testing Methods - Technical Section, Canadian Pulp and Paper Association, Montreal, Canada.

F. Duarte (1995), Optical Sensor for Pulp and Paper Dirt Counting (in Portuguese), Master Thesis, Faculdade de Ciências e Tecnologia da Universidade de Coimbra.

Jordan, D. B. and Nguyen. (1985) Dirt Counting with Microcomputers, Journal of Pulp and Paper Science, May, 73-77.

Peter F. M. N. (1995) Image Segmentation by Connectivity Preserving Relinking in Hierarchical Graph structures, Pattern Recognition, 6, 907-920. 